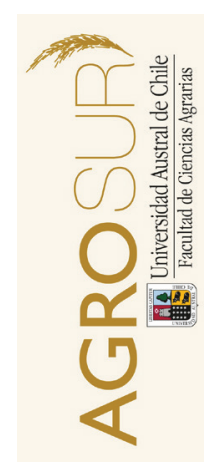

\title{
Potencial estimado de biogás en XIV Región a base de residuos urbanos y agroindustriales
}

\author{
Estimated biogas potential in XIV region based urban \\ and agro-biodegradable waste \\ Vermehren, M. $^{a *}$, Erlwein, A. $^{b, c}$ \\ ${ }^{a}$ Programa de Magíster en Ciencias Mención Producción Animal, Facultad de Ciencias Agrarias, \\ Universidad Austral de Chile. \\ ${ }^{b}$ Instituto de Ingeniería Agraria y Suelos, Facultad de Ciencias Agrarias, Universidad Austral de Chile, \\ Campus Isla Teja, Valdivia, Chile. \\ ${ }^{c}$ Centro Transdisciplinario de Estudios Ambientales CEAM-UACh.
}

\begin{tabular}{l} 
A R T I C L E IN F O \\
\hline Keywords: \\
Biogas \\
Renewable energy \\
Waste management
\end{tabular}

Original Research Article, Agriculture Engineering and Energy Management

*Corresponding author:

Martín Vermehren,

Alfredo Erlwein

E-mail address:

martinvermehrenparra@gmail.com,

aerlwein@uach.cl
A B S T R A C T

The lack of fossil fuels, high rates of growth in the national energy consumption, and environmental problems makes necessary to find new sources for clean and safe energy matrix.

This paper preliminarily estimated the potential of biogas production and its economic assessment in Los Rios Region, from urban and agro-biodegradable waste. For this, we quantified dairy waste, slaughterhouse sludge, water treatment sludge and household waste performing a compilation, standardization and processing available information. Then, using factors found in different databases, generate biogas, methane, electricity, heat and power load for the main plants of the region was estimated. Finding that the highest potential was in the dairy industry, using cheese whey. The slaughter industry had the lowest potential. The total potential for the region reached more than $25,500,000 \mathrm{~m}^{3}$ and would supply through cogeneration an electrical output of 9.0 MW. This energy could supply the electricity requirements of more than 170,000 people. Economic evaluation indicated feasibility for most of the projects evaluated. The economically feasible potential for the region reached 6.2 MWe.

\section{RESUMEN}

La falta de combustibles fósiles, las altas tasas de crecimiento en el consumo energético nacional y los problemas ambientales, hacen necesario buscar nuevas fuentes limpias y seguras para la matriz energética nacional.

En este trabajo se estimó en forma preliminar el potencial de producción de biogás y su evaluación económica, a partir de residuos biodegradables urbanos y agroindustriales de la Región de los Ríos. Para esto, se cuantificaron los residuos de industria láctea, matadero, lodos residuales líquidos y residuos domiciliarios (RSU) realizándose una recopilación, estandarización y procesamiento de la información disponible. Luego, utilizando factores encontrados en distintas bases de datos se estimó la generación de biogás, metano, energía eléctrica, térmica y potencia instalada para las principales plantas de la región, encontrándose que el mayor potencial se encuentra en la industria láctea, utilizando el suero del queso. La industria de mataderos tuvo el potencial más bajo. El potencial total para la región alcanzó más de $25.500 .000 \mathrm{~m}^{3}$ y surtiría mediante cogeneración una potencia eléctrica de 9,0 MW. Con esta energía se podría abastecer los requerimientos de electricidad de más de 170.000 personas.

La evaluación económica indicó viabilidad para gran parte de los proyectos evaluados. El potencial económicamente viable para la región alcanzó los 6,2 MWe.

Palabras clave: biogás, energía renovable, residuos.

\section{INTRODUCCION}

Mercado energético en Chile: Actualmente, el país importa casi las tres cuartas partes de la energía que consume. Entre los años 1990 y 2007 se evidenció un fuerte aumento en las importaciones de energía y un estancamiento de la producción local de ésta, lo que hace más vulnerable al país a condiciones externas. Es por ello que Chile hoy en día busca nuevas formas de aprovechar los recursos que posee para satisfacer la demanda creciente de energía (Soto y Werner, 2009). 
Por otra parte, el consumo global de energía en Chile durante los últimos 20 años se ha expandido a una tasa anual en torno al 5,6\%. En el mismo período, el aumento anual promedio del consumo de electricidad ha sido cerca de un 7,5\%. De acuerdo a estas cifras, el país ha debido duplicar su capacidad de suministro eléctrico aproximadamente cada 10 años (Zanelli et al., 2008).

Según CNE y GTZ (2009) la generación eléctrica total para el año 2007 fue de 55.914 GWh y provenía en un $38 \%$ de plantas hidroeléctricas, $10 \%$ de gas natural, $26 \%$ de carbón, $22 \%$ de petróleo combustible y un $3,1 \%$ de fuentes renovables no convencionales como pequeña hidráulica, biomasa y eólica.

Mercado eléctrico chileno: Según la Comisión Nacional de Energía, el mercado eléctrico en Chile se compone de 3 grandes áreas: generación, transmisión y distribución. Asimismo, el mercado eléctrico chileno se organiza en cuatro grandes sistemas eléctricos, éstos son: sistema interconectado del norte grande (SING), sistema interconectado central (SIC), sistema eléctrico de Aysén y sistema eléctrico de Magallanes (Hall et al., 2009).

La Región de Los Ríos se abastece de energía proveniente del sistema interconectado central, el cual abarca desde Tal-Tal, en la IV región, hasta Chiloé, en la X región.

El año 2010 la región de Los Ríos tuvo una generación de energía eléctrica de $771 \mathrm{GWh}$, representando tan solo un 1,28\% del total nacional (INE, 2011). El consumo eléctrico en la región para el mismo año fue del orden de los 552,77 GWh (INE, 2012), siendo éste inferior a la generación total en la región.

Por otra parte, el sistema eléctrico en Chile se encuentra altamente concentrado, es así como al año 2006 participaban un total de 70 empresas, de las cuales 28 eran generadoras, 5 transmisoras y 37 eran distribuidoras. En el caso de la generación, tres empresas (y sus filiales) poseían más del $89 \%$ de la potencia instalada en el SIC (51\% ENDESA, 20\% Colbún y 19\% AES Gener), otras doce empresas poseían el 10\% restante (CNE y GTZ, 2009).

Potencial energético: Las tecnologías disponibles en la actualidad permitirían a la región de Los Ríos aprovechar una gran variedad de fuentes energéticas, destacando un alto potencial hidroeléctrico seguido de un importante potencial en biomasa, y en menor medida, energía geotérmica, eólica y mini-hidráulica (Hall et al., 2009). Sumado a lo anterior, según (Monardez et al., 2008), la región posee un alto potencial en energía undimotriz y mareomotriz, puesto que sus costas son muy propicias para la generación eléctrica debido a su reducida variabilidad estacional, baja dispersión direccional y altos períodos. Además, existe un proyecto ya aprobado de gasificación de carbón en la localidad de Mulpún (Chile, Servicio de evaluación de impacto ambiental (SEIA), 2012), el que podría aportar energía al SIC.
Biogás: Dentro de las energías provenientes de biomasa, el biogás se presenta como una alternativa para la región (Hall et al., 2009). Flotats et al., (1997) lo definen como un biocombustible gaseoso que se produce por la descomposición anaerobia (en ausencia total de oxigeno o nitratos) de la materia orgánica, resultando una mezcla de gases de proporción variable. Eltawil y Belal (2009) indican que biogás producido a partir de residuos agrícolas presenta entre un 54 a $75 \%$ de $\mathrm{CH}_{4}$ (metano, gas que le otorga las propiedades combustibles a la mezcla), de un 33 a un $38 \%$ de $\mathrm{CO}_{2}$, menos de un $2 \%$ corresponde a $\mathrm{O}_{2}$ y $\mathrm{N}_{2}$ y hasta $2622 \mathrm{ppm}$ pueden corresponder a $\mathrm{H}_{2} \mathrm{~S}$. En cuanto a la potencia calorífica, para el mismo tipo de residuos señalan que se encuentra entre los 7.476 y $6.658 \mathrm{kcal} \mathrm{m}^{-3}$. Las instalaciones especialmente diseñadas para optimizar este proceso se designan como "digestores de metano", "plantas de biogás", "biodigestores" o simplemente "reactores anaerobios" (Flotats et al., 1997).

Por otra parte, la utilización de residuos orgánicos para la producción de energía supone muchas ventajas económicas, ambientales y sociales. El proceso fermentativo reduce la carga patógena de los residuos, especialmente los de producción animal o centros urbanos, constituyendo una alternativa para el tratamiento de ellos. Según Martin (2007) la fermentación reduce los niveles de patógenos tales como Salmonella spp. Escherichia coli (Migula) Castellani \& Chalmers, virus enteríticos y huevos fértiles de Helmintos. Côté et al. (2006), citado por Massé et al. (2008) señala que procesos fermentativos remueven poblaciones de Salmonella spp., Cryptosporidium y Giardia, y reduce poblaciones de microorganismos indicadores como Escherichia coli y coliformes desde un $97,94 \%$ a un 100\%. Martin et al., (2003) citado por Martin et al. (2012) reportaron una reducción media de 99\% para Mycobacterium avium paratuberculosis luego de una digestión anaerobia.

En vista de la problemática presentada y la oportunidad existente, el presente trabajo tiene como objetivo estimar en forma preliminar el volumen de biogás posible de producir en la Región de los Ríos y su evaluación económica a partir de los principales residuos orgánicos biofermentables urbanos y agroindustriales

\section{MATERIAL Y MÉTODO}

\section{Material}

Se utilizaron bases de datos científicas Science Direct y Web of Science (ISI), como también otras disponibles en el sistema de biblioteca electrónica de la Universidad Austral de Chile. También se recurrió a plataformas gubernamentales nacionales como Instituto Nacional de Estadística (INE), Oficina de Estudios de Políticas Agrarias (ODEPA), Comisión Nacional de Energía (CNE), Superintendencia de Servicios Sanita- 
rios (SISS), Servicio de Evaluación de Impacto Ambiental (SEIA) y Ministerio de Energía.

Para desarrollar los cálculos, gráficos y cuadros se utilizó el programa computacional Microsoft Excel $2010^{\circledR}$.

\section{Método}

El método para cuantificar los distintos residuos y producción total de biogás y metano varió según cada residuo, como se indica a continuación:

\section{Residuos orgánicos domiciliarios y municipales}

Se cuantificaron a nivel de vertedero o relleno sanitario por comuna, a excepción del vertedero de la comuna de Valdivia, que funciona como lugar de acopio final para varias comunas de la región. Según lo informado por Asociación de Municipalidades de los Ríos para el año 2011 se obtuvo el total de residuos generados en la región y la cantidad de materia orgánica presente en éstos. Utilizando los factores de metanización que mencionan CNE y GTZ (2007), se calculó el potencial total de biogás y metano.

\section{Residuos orgánicos industriales}

Se realizó un catastro general a nivel regional en donde se cuantificó la generación de residuos de la industria láctea, de matadero, cervecería y pisciculturas, seleccionando finalmente solo los dos primeros, por ser significativamente más relevantes que los demás.

\section{Industria láctea}

De la totalidad de los productos lácteos que se elaboran en la región de los Ríos, Zaror (2000) indica que la producción de queso es la única que genera un residuo (o subproducto, según el uso que se le dé) de alta carga orgánica. Por tanto, en este trabajo se asumió que la mayor parte de éstos corresponden a lacto suero, despreciando los residuos provenientes del lavado de estanques, derrames y otros. De esta forma, según el promedio de la producción regional de queso entre los años 2011 y 2012 con datos de ODEPA y la cantidad de suero generado por unidad de queso producido, se estimó la generación anual basada en lacto suero para la región de los Ríos, considerando los valores presentados por Prazeres et al. (2012) respecto a generación de suero por unidad de queso producido. Luego, con los valores que muestran Comino et al. (2012) se estimó el volumen de biogás y metano posible de producir en la región.

El cálculo anterior se complementó considerando producción regional por año de los principales productos lácteos, con datos de ODEPA y generación media de solidos suspendidos por unidad de producto produ- cido, información que presenta detalladamente Zaror (2000). De esa manera, se obtiene la generación anual de solidos suspendidos provenientes de la industria láctea, a nivel regional.

\section{Industria de matadero}

Se evaluaron los 2 mataderos autorizados para la región: Frigorífico Valdivia y Frigorífico Balmaceda (en adelante FRIVAL y FRIGOBAL respectivamente). Para estimar los residuos de ambos se utilizó como base lo expuesto en la declaración de impacto ambiental de la planta FRIVAL presentado en el servicio de evaluación ambiental (SEIA, 2005) en donde se caracterizan sus RILES. El volumen de éstos se obtuvo del trabajo presentado por Altaner (2009) en donde indica el flujo diario de residuos líquidos generados en la planta FRIVAL, obteniéndose de esa forma la cantidad total de residuos sólidos generados en 1 año, asumiendo un funcionamiento de la planta durante los 365 días del año. El flujo de residuos anuales para la planta FRIGOBAL se encuentra disponible en la superintendencia de Servicios Sanitarios (de ahora en adelante SISS). La caracterización de estos residuos no se encontró disponible para FRIGOBAL, por lo tanto se asumió que sus residuos son de igual composición a los de FRIVAL, de modo que se utilizó como base la misma información utilizada para esa planta.

Puesto que según lo señalado en la declaración de impacto ambiental (SEIA, 2005), las grasas son separadas de los RILES, para el cálculo total de biogás y metano se analizaron por separado el potencial de las grasas y el potencial del resto del RIL, utilizando para las grasas los factores señalados por Institut für Energetik und Umwelt (IEU, 2006) y para el RIL los señalados por Iglinski et al. (2012).

\section{Lodos residuales líquidos}

Se consideraron a partir de plantas de tratamiento de aguas servidas (PTAS). La información sobre lodo deshidratado en base seca generado por planta para el año 2012 se obtuvo a través de la SISS. Antes del año 2012 sólo se registraba el volumen de lodo generado por planta, sin tener información del contenido de humedad ni la densidad. A partir del año 2012 se comenzó a registrar el lodo generado en base seca, por lo tanto, se utilizó esta última información para los cálculos. Al comparar los volúmenes del año 2012 con los de años anteriores se verificó que había consistencia, lo que mejora la confiabilidad de los valores, considerando que se utilizó sólo un año para los cálculos. Para estimar el biogás y metano generado se utilizó un promedio del rango de metanización presentado por Deublein y Steinhauser (2008), el cual es coherente con lo expresado por CNE y GTZ (2007) y Kaltschmitt et al. (2013). 


\section{Transformaciones energéticas}

Una vez obtenido el potencial de biogás y metano para cada residuo, se calculó la cantidad de energía eléctrica considerando que $1 \mathrm{~m}^{3}$ de metano tiene 9.464 kcal. Una vez obtenido el contenido calórico del biogás, se transformó a energía eléctrica considerando $1 \mathrm{kcal}=$ 0,001163 kWh (Quesada et al., 2007).

Se consideró para la transformación la cogeneración con un motor CAT 3520C de una eficiencia eléctrica de un 38\% y térmica de un 40\% (CNE y GTZ, 2007). La potencia eléctrica se calculó suponiendo un funcionamiento del motor de 7.784 horas en el año y a potencia constante (CNE y GIZ, 2012).

\section{Evaluación económica}

Para el cálculo económico se elaboró un cuadro en donde se estima una inversión inicial según potencia y costos de mantención y operación para cada planta, considerando datos de CNE y GTZ (2007) y Gamma Ingenieros (2011). Con esta información se asignó una inversión para cada proyecto en particular, los cuales se definieron considerando las plantas que en la actualidad generan residuos.

Los ingresos se cuantificaron considerando la venta de energía eléctrica, potencia eléctrica y energía térmica producida. No se consideraron ingresos por concepto de bonos de carbono, biofertilizante y otros beneficios ambientales, puesto que no se encontró un método cuantitativo simple, adecuado a los alcances de esta investigación. Para la energía eléctrica, el precio a utilizar corresponde al precio nudo medio de mercado para el sistema interconectado central en el período correspondiente septiembre 2012 a diciembre 2012 (CNE, 2013) fijado en octubre del 2012 según la Comisión Nacional de Energía, valor que alcanzó en ese período los $\$ 54.488$ por MWh. La potencia eléctrica se valorizó en US\$ 8,784 por $\mathrm{kWh}^{\mathrm{mes}}{ }^{-1}$ de acuerdo a lo expresado por el Ministerio de Energía publicado en el diario oficial el día 12 de febrero del 2013. Para cuantificar los ingresos por venta de energía térmica se consideró la energía térmica generada ( $\mathrm{MWh}$ ) por planta y a ésta se le asignó un valor de 0,8 UF por MWh térmico, equivalente a lo que cuesta generar esa cantidad de energía a partir de chips de madera según lo señalado por Pavez (2013). Este valor, según el mismo autor, está muy por debajo de lo que cuesta generar la misma cantidad de energía a partir de fuentes como el petróleo diésel, por tanto, se consideró correcto para ser utilizado en esta evaluación sin sobre estimar estos ingresos. El valor de UF utilizado fue el alcanzado al día 18 de junio del 2013, de $\$ 22.852,67$. Como criterio de evaluación económica se utilizó el método del valor actual de beneficios netos (de ahora en adelante VABN), por ser un método de amplio uso en evaluaciones eco- nómicas (Altuve, 2004), y consiste en el valor que tienen en un momento dado los pagos y cobros realizados y los que se realizarán en el futuro, de forma de determinar si una inversión llevada a cabo en el presente se verá compensada por el valor de los pagos y cobros en el futuro (Gil y Celma, 2003).

\section{RESULTADOS Y DISCUSIÓN}

\section{Residuos orgánicos domiciliarios y municipales}

Las comunas de Valdivia, Panguipulli, Lanco, San José, Máfil, Los Lagos y Corral envían sus residuos al vertedero Morrompulli, ubicado en la comuna de Valdivia, por lo tanto, por ser lugar de acopio final se consideró este vertedero y los de las restantes comunas de la región (Futrono, La Unión, Río Bueno, Lago Ranco y Paillaco), según la información entregada por la Asociación de Municipalidades de Los Ríos, tal como lo indica el cuadro 1.

Según la asociación de Municipalidades de Los Ríos, la proporción de materia orgánica presente en los residuos recibidos en el vertedero Morrompulli promedia el 55\% del total de éstos, en peso húmedo, asumiéndose el mismo valor para los otros vertederos. Para el cálculo de biogás y metano generado al año, se utilizaron valores de $60 \mathrm{~m}^{3}$ ton $^{-1} \mathrm{MOH}$ con un $50 \%$ de metano, considerándose la generación de biogás por tonelada de materia orgánica húmeda contenida en el residuo. Con estos datos, se calculó el potencial de biogás y metano para la Región de los Ríos, mostrándose los resultados en el cuadro 1. Como se observa en éste, el mayor potencial se encuentra en el vertedero Morrompulli, lo que se explica principalmente por la mayor población que cubre, y por consiguiente, el mayor tamaño de éste.

Es importante señalar que para estimar este potencial, se consideraron los residuos tal como llegan a vertedero, esto es, materia orgánica de composición y humedad variable, mezclada con metales, plásticos, vidrio, pilas, entre varios otros componentes de los RSU. Además, la mayor parte de los residuos envuelto por bolsas de polietileno u otro polímero de lenta degradación. El potencial podría ser considerablemente mayor si se separase lo orgánico del resto de residuos. De esta forma, IEU (2006) señala que por tonelada de materia húmeda de restos de comida, se pueden producir hasta $480 \mathrm{~m}^{3} \mathrm{de}$ biogás y hasta $200 \mathrm{~m}^{3}$ a partir de residuos verdes. En este trabajo, se estimaron para la región 75.332 ton de materia orgánica húmeda. Lo que podría generar, en el caso que se encuentren separados del resto de los residuos, entre 15.066 .400 y $36.159 .360 \mathrm{~m}^{3}$ de biogás al año, lo que equivale a entre 4 y 9 veces lo estimado en este trabajo.

Cabe destacar también, que sólo se consideraron residuos de áreas cubiertas por el servicio de recolección de basura, esto es cercano al $100 \%$ de la población urbana pero sólo cerca de un 50\% de la rural, según 
estimaciones del Gobierno Regional para el año 2012. Por lo tanto, los residuos de un aproximado de 60.000 personas (de un total de 360.000) no fueron consideradas en el cálculo del potencial regional.

\section{Industria láctea}

Se consideró la producción anual de queso para las 5 mayores plantas de este producto en la región (ODEPA, 2013). Segmento que es denominado por este organismo como industria láctea mayor. Estas plantas son:

- Planta Colún, comuna de La Unión

- Planta Soprole, comuna de Los Lagos

- Planta Lácteos Las Parcelas, comuna de Valdivia.

- Planta Lácteos del Sur S.A., comuna de Río Bueno

- Planta Quillayes - Peteroa, comuna de Futrono
Además, se consideró el total de la producción de quesos en la región (INE, 2013), a menor escala, denominada por INE como "láctea menor".

De la elaboración de queso se genera suero de alta carga orgánica (Zaror, 2000) compuesto principalmente por agua, proteínas y lactosa (Santos, 1983). Los valores utilizados fueron: 6\% de materia seca (Santos, 1983), $80 \%$ de MO (Comino et al., 2012) y 9 kg de suero húmedo por kg de queso producido (Prazeres et al., 2012). Para los cálculos se promediaron valores de industria láctea mayor y menor. Resultados en el cuadro 2.

Estos valores dan cuenta del gran potencial de la industria láctea mayor, principalmente de las plantas Colún y Soprole, las que debido a su elevada producción serían responsables de cerca del $75 \%$ del biogás que se podría producir en la Región.

Cuadro 1. Generación anual de residuos y volumen anual de biogás y metano posible de producir por vertedero en la Región de Los Ríos a partir de residuos domiciliarios y municipales.

Table 1. Annual waste generation rate and annual biogas and methane volume possible to generate per landfill in Los Rios region from household and municipal waste.

\begin{tabular}{lccc}
\hline Vertedero & Residuos recibidos $^{*}\left(\right.$ ton $\left.\mathbf{~ a n ̃ o ~}^{-1}\right)$ & Biogás $\left(\mathbf{m}^{\mathbf{3}} \mathbf{a n ̃ o}^{-\mathbf{1}}\right)$ & Metano $_{\left(\mathbf{m}^{\mathbf{3}} \mathbf{a n ̃ o}^{-\mathbf{1}}\right)}$ \\
\hline Morrompulli (Valdivia) & 111.167 & 3.668 .524 & 1.834 .262 \\
Futrono & 2.300 & 75.900 & 37.950 \\
La Unión & 12.000 & 396.000 & 198.000 \\
Lago Ranco & 1.800 & 59.400 & 29.700 \\
Paillaco & 3.200 & 105.600 & 52.800 \\
Río Bueno & 6.500 & 214.500 & 107.250 \\
\hline TOTAL & 136.967 & 4.519 .924 & 2.259 .962 \\
\hline
\end{tabular}

*Fuente: Asociación de Municipalidades de los Ríos.

Cuadro 2. Generación anual de suero seco, materia orgánica y producción estimada de biogás y metano para las industrias lácteas de la región de los Ríos.

Table 2. Annual generation rate of dried whey, organic matter and estimate biogas and methane production from dairy industry in Los Rios region.

\begin{tabular}{|c|c|c|c|c|}
\hline Industria & Suero seco $(\mathrm{kg})$ & Materia orgánica (ton MS) & Biogás $\left(m^{3}\right)$ & Metano $\left(\mathrm{m}^{3}\right)$ \\
\hline Láctea mayor & 26.129 .210 & 22.863 & 20.005 .176 & 14.003 .623 \\
\hline Colún & 12.710 .821 & 11.122 & 9.731 .722 & 6.812 .206 \\
\hline Soprole & 8.351.214 & 7.307 & 6.393 .898 & 4.475 .729 \\
\hline Lácteos Las Parcelas & 2.474 .893 & 2.166 & 1.894 .840 & 1.326.388 \\
\hline Lácteos del Sur & 1.854 .595 & 1.623 & 1.419 .924 & 993.947 \\
\hline Quillayes - Peteroa & 737.687 & 646 & 564.792 & 395.354 \\
\hline Láctea menor & 241.344 & 211 & 184.771 & 129.340 \\
\hline TOTAL REGION & 26.370 .544 & 23.074 & 20.189 .947 & 14.132 .963 \\
\hline
\end{tabular}




\section{Industria de matadero}

En la región actualmente existen 2 mataderos autorizados: FRIVAL y FRIGOBAL, por lo tanto, para aprovechar el agrupamiento de éstos residuos y facilitar la cuantificación de éstos, sólo se consideraron ambas plantas, dejando de lado otras fuentes de generación de residuos, tales como mataderos no registrados o faenamientos realizados en sectores rurales.

Para obtener la información correspondiente a la planta FRIVAL se consideró la caracterización de los RILes presentada en el SEIA (2005) por representante de la planta. El volumen de RILes diario generado en la planta lo indica Altaner (2009) en $650 \mathrm{~m}^{3}$, obteniéndose de ésta forma los datos presentados en el cuadro 3.

Cabe destacar que los valores de sólidos suspendidos sumados a los de aceites y grasas, son significativamente inferiores a los de la $\mathrm{DBO}_{5}$, sin embargo, no se encontraron indicadores adecuados para transformar esta última variable en biogás, por lo tanto, se utilizaron los sólidos suspendidos y las grasas para esto, de modo que es posible una subestimación del potencial para esta industria.

Para estimar los residuos generados en la planta FRIGOBAL se consideró el volumen de RILes generados, según lo señalado por la Superintendencia de Servicios Sanitarios, información que entregó a este organismo la planta de tratamiento de aguas residuales de la empresa Essal para el año 2011, en la comuna de La Unión. Esta empresa sanitaria mantiene contrato con FRIGOBAL para tratar sus RILes. Por este motivo, el frigorífico no fue considerado en el potencial regional de biogás, puesto que está incluida en esa planta de tratamiento de aguas servidas. Sin embargo, se estimó el potencial de este frigorífico para obtener una estimación del potencial regional de la industria de matadero. A continuación, se muestra el potencial regional de la industria de matadero.

En comparación a la industria láctea, el potencial de la industria de mataderos es significativamente inferior, representando menos del $2 \%$ del potencial regional de residuos industriales. Esto probablemente se debe a que los residuos que se consideraron para la producción de biogás, a diferencia del lacto suero, son de menor contenido energético, puesto que los RILes utilizados son una mezcla de 2 tipos de RILes: aguas rojas y aguas verdes. Las primeras corresponden al agua-sangre proveniente de la planta de faena; aguas de lavado de los equipos, productos y pisos, lavado de los equipos de la sala de procesamiento de vísceras rojas, zona limpia de procesamiento de guatitas y tripas, decomisos y desposte. Las segundas corresponden a aguas provenientes del lavado de camiones, corrales y salas de vísceras verdes, salas de procesamiento de vísceras verdes y pisos de las salas de zona sucia del procesamiento de vísceras verdes, aguas del lavado de equipos. Extracción del contenido ruminal de estómagos, vómito. Por lo tanto, no se considera en el cálculo el grueso de las grasas, restos cárnicos, sangre o huesos, puesto que actualmente son separados y comercializados en el mercado. En eventualidad que el precio de la energía aumenta, podrían considerarse estos residuos para producción de biogás y de ésa forma, aumentar la generación eléctrica y el potencial instalado total.

Cuadro 3. Tasa anual de generación de materia orgánica, biogás y metano en la planta FRIVAL.

Table 3. Annual generation rate of organic matter, biogas and methane in FRIVAL industry.

\begin{tabular}{|c|c|c|c|}
\hline Parámetro & Materia orgánica (ton año ${ }^{-1}$ ) & Biogás (m² año $\left.{ }^{-1}\right)$ & Metano $\left(\mathrm{m}^{3}\right.$ año $\left.^{-1}\right)$ \\
\hline Sólidos Suspendidos & 370 & 174.331 & 108.957 \\
\hline Aceites y Grasas & 125 & 87.329 & 54.581 \\
\hline TOTAL & 495 & 261.660 & 163.538 \\
\hline
\end{tabular}

Cuadro 4. Tasa de generación anual de residuos orgánicos, biogás y metano para la industria de matadero.

Table 4. Annual generation rate of organic waste, biogas and methane for slaughterhouse industry.

\begin{tabular}{lccc}
\hline Planta & Materia orgánica (ton $\left.\mathbf{a n ̃ o}^{-1}\right)$ & Biogás $\left(\mathbf{m}^{\mathbf{3}} \mathbf{a n ̃ o}^{-\mathbf{1}}\right)$ & \left.${\text { Metano }\left(\mathbf{m}^{\mathbf{3}} \mathbf{a n ̃ o}\right.}^{-\mathbf{1}}\right)$ \\
\hline FRIVAL & 495 & 261.660 & 163.538 \\
FRIGOBAL & 25 & 13.269 & 8.448 \\
\hline TOTAL & 520 & 274.929 & 171.986 \\
\hline
\end{tabular}




\section{Lodos residuales líquidos}

Se evaluaron las 11 plantas de tratamiento de aguas servidas (PTAS) que tratan las aguas residuales de las principales ciudades de la región. Las comunas de La Unión y Río Bueno comparten una sola planta, ubicada en esta última. Con la generación mensual de lodo (en toneladas de lodo deshidratado, como también la generación mensual en volumen durante los años 2010 y 2011 para cada planta, usando esta última información a modo de comparación, para fortalecer la información) presentada por la SISS (2013), considerando un $60 \%$ de contenido orgánico en la materia seca del residuo (Schievano et al., 2009) y un rendimiento de biogás de $475 \mathrm{~m}^{3}$ por tonelada de MS orgánica, según Deublein y Steinhauser (2008), se obtuvieron los resultados que muestra el cuadro 5 .

El potencial de biogás de estos residuos es considerablemente inferior al de los residuos urbanos domiciliarios, lo que se puede explicar, entre otras cosas, porque el residuo considerado para el cálculo corresponde al lodo obtenido luego de un tratamiento aerobio, siendo el de lodos activados el sistema utilizado en casi la totalidad de las plantas de la región, exceptuando la planta Valdivia de la empresa Aguas Décima.

Actualmente no se han desarrollado proyectos en la región para tratar las aguas residuales con una digestión anaerobia. Según CNE y GTZ (2007), es posible realizar un pre tratamiento anaerobio con obtención de biogás aprovechando cerca de un 30\% de la energía presente en el residuo, además de reducir el consumo energético requerido en el tratamiento aerobio. De esta forma, el potencial de biogás a partir de residuos de plantas de tratamiento de aguas servidas podría aumentar de 852.213 $\mathrm{m}^{3}$ a un rango de entre $1.990 .000 \mathrm{~m}^{3}$ y $3.970 .000 \mathrm{~m}^{3}$.

Por otra parte, las diferencias encontradas entre las plantas de la empresa Essal y la planta Valdivia perteneciente a la empresa Aguas Décima se explican porque esta última, además de cubrir una mayor población, realiza principalmente tratamiento primario con desinfección, generando mayor cantidad de lodos que plantas de Essal, en donde se realiza tratamiento completo de lodos activados.

\section{Generación eléctrica, térmica y capacidad instalada}

Como era de esperar, el mayor potencial, con valores muy superiores, lo presentan los residuos industriales, particularmente la industria láctea con un uso potencial del suero para fines energéticos.

Como se aprecia en el cuadro 6, la potencia instalada eléctrica en la región alcanzaría los 9,0 MW.

\section{Evaluación económica}

La evaluación se realizó únicamente a plantas donde actualmente existen residuos agrupados, no considerando costos de recolección y transporte. En cuanto

Cuadro 5. Tasa de generación anual de lodo deshidratado generado (ton año-1 ${ }^{-1}$, volumen de biogás y metano posible de producir en la región de los ríos por año para cada planta.

Table 5. Annual generation rate of dewatered ludge (ton year ${ }^{-1}$ ), biogas and methane volume possible to produce in Los Rios region for each plant.

\begin{tabular}{|c|c|c|c|}
\hline Planta tratamiento aguas servidas & Lodo deshidratado (ton año-1) & Biogás $\left(m^{3} a \tilde{a n} 0^{-1}\right)$ & Metano $\left(m^{3} a_{\tilde{0}} o^{-1}\right)$ \\
\hline Corral & 3,1 & 871 & 540 \\
\hline Futrono & 56,0 & 15.959 & 9.894 \\
\hline Lago Ranco & 3,0 & 847 & 525 \\
\hline Lanco & 99,3 & 28.310 & 17.552 \\
\hline Los Lagos & 86,3 & 24.587 & 15.244 \\
\hline Máfil & 47,9 & 13.657 & 8.468 \\
\hline Paillaco & 70,1 & 19.991 & 12.395 \\
\hline Panguipulli & 98,1 & 27.947 & 17.327 \\
\hline Río Bueno & 660,9 & 188.346 & 116.775 \\
\hline S. J. de la Mariquina & 39,6 & 11.276 & 6.991 \\
\hline Valdivia & $1.826,0$ & 520.421 & 322.661 \\
\hline TOTAL GENERAL & $2.990,2$ & 852.213 & 528.372 \\
\hline
\end{tabular}


Cuadro 6. Generación anual de residuos, potencial eléctrico, térmico y potencia instalada eléctrica para las distintas fuentes, y el total de la región de los Ríos.

Table 6. Annual generation of waste, electrical and thermal potential, and electrical installed power for different sources, and the total of Los Rios region.

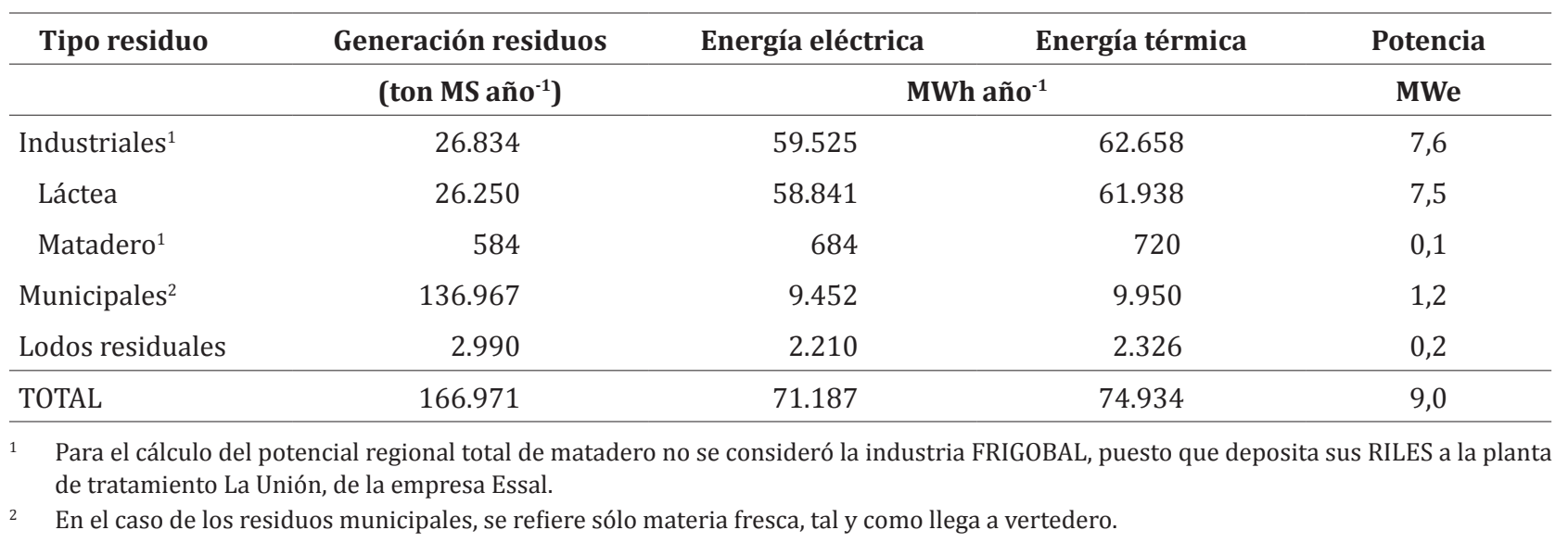

a las plantas de tratamiento de aguas servidas de la región, se consideraron únicamente las que poseen una potencia instalada estimada superior a $10 \mathrm{~kW}$.

Para estimar la inversión inicial se consideró el costo del motor de cogeneración, costo de conexión a la red eléctrica, costo del digestor y equipos de la planta, costo de edificación y costos de conexión a la red de vapor, según lo señalado por GTZ y CNE (2007). Respecto a los costos de operación, se consideraron costos de mantenimiento del motor, mantenimiento de equipos, costos de personal, vida operativa, interés anual y costos de capital, también según GTZ y CNE (2007), todo esto para proyectos con una potencia instalada superior a $0,3 \mathrm{MW}$, y para proyectos con potencia instalada entre 0,05 y 0,2 MW se consideró la información presentada por Gamma Ingenieros (2011). La razón de la diferencia en la inversión señalada por estos dos autores es justificada por el menor costo de los distintos materiales para la construcción en proyectos de diferentes escalas.

A continuación se presentan los resultados del análisis económico de tipo VABN para los proyectos de potencias instaladas superiores a $10 \mathrm{~kW}$.

\section{Residuos domiciliarios y municipales}

En estos residuos, por estar actualmente los rellenos sanitarios o vertederos autorizados en lugares alejados de centros productivos, condición que dificulta la venta de energía térmica, se evaluaron 2 escenarios; uno considerando la venta de esta energía y la otra sin considerarla, no habiendo cambios en la rentabilidad de cada proyecto entre uno y otro escenario. A continuación, en el cuadro 7 se presentan los resultados del VABN.

A pesar de que el relleno sanitario Morrompulli mostró ser el que maneja mayores volúmenes de resi- duos, no logró un valor positivo en el análisis. Esto puede explicarse por la alta inversión relativa (US\$ kWe instalado ${ }^{-1}$ ) que se mostró en el cuadro 7. Por lo tanto, se lograron índices positivos tan solo en los vertederos de las comunas de La Unión y Río Bueno.

\section{Evaluación económica para industria láctea}

Los resultados del análisis de VABN para las plantas de la industria láctea mayor se presenta en el cuadro 8.

Como era esperable, las 2 más grandes plantas lácteas de la región mostraron índices económicos positivos. Sin embargo, Lácteos Las Parcelas y Lácteos del Sur arrojaron indicadores negativos, posiblemente por su menor capacidad de producción. La planta Quillayes - Peteroa arrojó indicadores positivos. Lo anterior se puede explicar por qué según Gamma Ingenieros (2011) proyectos con potencia similar o menor a $500 \mathrm{~kW}$ requieren alta inversión relativa (US\$ $\mathrm{kW}$ instalado-1) haciendo inviable dicha tecnología en el país. Sin embargo, plantas con potencias cercanas o menores a $100 \mathrm{~kW}$ instalados pueden abaratar los costos de inversión al reducir costos de materiales y tecnologías como piscinas cubiertas o estanques de fibra de vidrio enterrados, sin agitación ni control de temperatura. Con dichos cambios se disminuye la eficiencia en la obtención de biogás, sin embargo, se produce un importante ahorro en la inversión.

\section{Evaluación económica para industria de matadero}

Como se señaló anteriormente, se evaluó solo la planta FRIVAL, excluyendo la planta FRIGOBAL por tener una potencia instalada menor a $10 \mathrm{~kW}$.

Como se aprecia en el cuadro 9, la evaluación de esta planta arrojó indicadores positivos, resultando 
Cuadro 7. VABN para cada proyecto de vertederos en la región. La columna 1 considera la venta de energía térmica y la 2 no lo considera para la evaluación.

Table 7. NPV for each landfill project in the region. Column 1 considers sale of thermal energy and column 2 is not considered for evaluation.

\begin{tabular}{lccc}
\hline \multicolumn{1}{c}{ Vertedero } & Potencia eléctrica $(\mathbf{M W})$ & \multicolumn{1}{c}{ VABN $^{1}$} & VABN $^{2}$ \\
\hline Morrompulli & 0,97 & $-\$ 538.162$ & $-\$ 1.123 .496 .252$ \\
Futrono & 0,02 & $-\$ 44.609 .306$ & $-\$ 67.842 .772$ \\
La Unión & 0,11 & $\$ 363.029 .458$ & $\$ 241.811 .371$ \\
Lago Ranco & 0,02 & $-\$ 65.621 .613$ & $-\$ 83.804 .326$ \\
Paillaco & 0,03 & $-\$ 6.787 .152$ & $-\$ 39.111 .975$ \\
Río Bueno & 0,06 & $\$ 131.894 .076$ & $\$ 66.234 .280$ \\
\hline
\end{tabular}

TOTAL REGIÓN 1,20

${ }^{1}$ Considera venta de energía térmica

${ }^{2}$ No considera venta de energía térmica

viable económicamente. Lo que se explicaría también por su bajo costo de inversión, como lo señala Gamma Ingenieros (2011).

\section{Evaluación económica para lodos residuales}

De las 11 plantas existentes en la región, se evaluaron únicamente los 2 proyectos con potencia instalada superior a $10 \mathrm{~kW}$, mostrándose los resultados en el cuadro 10:

El análisis arrojó inviabilidad económica para la planta Río Bueno. Sin embargo, el proyecto Valdivia, de la planta del mismo nombre, perteneciente a la empresa Aguas Décima, mostró indicadores positivos.

Cabe destacar que la evaluación económica realizada a escala regional es preliminar, sin profundizar mayormente en las condiciones específicas de cada proyecto, por lo que se requieren más estudios para profundizar lo visto en este trabajo. Por otra parte, corresponde a una evaluación económica tradicional, en donde solo se consideran variables que tienen un valor monetario transable en el mercado, no considerando otros como los impactos ambientales involucrados. El aprovechamiento de residuos para obtención de biogás permite una reducción volumétrica de éstos, con un bajo costo energético (contrariamente a lo que ocurre por ejemplo con las plantas de tratamiento aerobio de aguas servidas, las que requieren de energía para agitadores y otros procesos), efectivo tratamiento natural contra varios agentes patógenos y además se evita la liberación de metano a la atmósfera, muy importante considerando un contexto de cambio climático. En esa misma línea, el obtener electricidad a partir de estos residuos reduce la utilización de otros combustibles de mayor impacto ambiental, como los combustibles fósiles o la leña, mejorando la calidad del aire, reduciendo
Cuadro 8. VABN para cada planta de industria láctea mayor en la región.

Table 8. NPV for each dairy plant in the region.

\begin{tabular}{lcc}
\hline \multicolumn{1}{c}{ Planta } & Potencia (MWe) & VABN \\
\hline Colún & 3,6 & $\$ 8.187 .078 .899$ \\
Soprole & 2,4 & $\$ 4.870 .682 .997$ \\
Las Parcelas & 0,7 & $-\$ 1.294 .065 .626$ \\
Lácteos del Sur & 0,5 & $-\$ 824.055 .920$ \\
Quillayes - Peteroa & 0,2 & $\$ 865.680 .171$ \\
\hline TOTAL & 7,4 & \\
\hline
\end{tabular}

Cuadro 9. VABN para planta FRIVAL de industria de matadero en la región.

Table 9. NPV for FRIVAL plant from slaughterhouse industry

\begin{tabular}{ccc}
\hline Planta & Potencia (MWe) & VABN \\
\hline FRIVAL & 0,087 & $\$ 275.255 .602$ \\
\hline
\end{tabular}

Cuadro 10.VABN para las plantas de tratamiento de aguas servidas de la región con potencias instaladas superiores a $10 \mathrm{~kW}$.

Table 10. NPV for each plant of wastewater treatment in the region with installed power greater than $10 \mathrm{~kW}$.

\begin{tabular}{lcc}
\hline \multicolumn{1}{c}{ Planta } & Potencia (MWe) & VABN \\
\hline Río Bueno & 0,072 & $-\$ 31.030 .210$ \\
Valdivia & 0,198 & $\$ 163.327 .483$ \\
\hline TOTAL REGIONAL & 0,325 & \\
\hline
\end{tabular}


presión por uso del bosque y mitigando los efectos del cambio climático al reducir el metano que se libera a la atmosfera. En cuanto a los impactos sociales, el desarrollo de proyectos de este tipo podría aumentar la tasa de ocupación al haber una empleabilidad asociada a cada proyecto. Por lo tanto, un análisis completo debiera considerar los factores anteriormente considerados.

El potencial total de biogás en la región alcanzaría los $25.600 .000 \mathrm{~m}^{3}$, lo que representaría un $12,8 \%$ del total de biogás que era producido a nivel nacional para el 2010 (CNE y Gamma Ingenieros, 2010), y permitiría instalar una potencia eléctrica cercana a 9,0 MW. Ésta potencia representa cerca del $17 \%$ de la potencia eléctrica total instalada en el país a julio del año 2016 (CIFES, 2016).

En cuanto a proyectos de biogás a nivel nacional, según CNE y Gamma Ingenieros (2010), para el año 2012 existían un total de 20 plantas funcionando, que sumadas producían cerca de 200 millones $\mathrm{de}^{3}$ de biogás. Sin embargo, solo aproximadamente 35 millones $(17,5 \%)$ eran aprovechados, el resto se quemaban en antorchas. Esta tendencia ha comenzado a cambiar y actualmente ya hay más de 10 proyectos a nivel nacional que contemplan el aprovechamiento energético mediante transformación a energía eléctrica, térmica o ambas.

En cuanto a la generación eléctrica, con el biogás producido a partir de los residuos considerados en este trabajo, se podrían generar 71.187 MWh al año, lo que corresponde a un 7,6 \% del total generado en la región el año 2015 (INE, 2015). Además, a nivel de consumo por habitante, según INE (2008), para ese año, el consumo promedio de electricidad entre las VIII y XII regiones bordeaba los $416 \mathrm{kWh}$ anual. La generación total eléctrica por año, estimada en este trabajo, alcanza los $71.187 \mathrm{MWh}$, por tanto, únicamente a partir del biogás obtenido de residuos actualmente generados por actividades urbanas e industriales a gran escala se podría abastecer la demanda eléctrica de al menos 170.000 personas, población superior a la reportada para la ciudad de Valdivia en el censo 2012.

\section{CONCLUSIONES}

En la Región de los Ríos actualmente se generan en promedio 136.967 ton año ${ }^{-1}$ de residuos domiciliarios, de las cuales 75.332 ton año ${ }^{-1}$ son orgánicas.

Los lodos de plantas de tratamiento de aguas servidas generados a nivel regional alcanzan en promedio las 2.990 ton MS año-1.

Los principales grupos de residuos industriales orgánicos generados en la región corresponden a los de la industria láctea e industria de matadero, generando 26.250 y 584 ton MS año ${ }^{-1}$ respectivamente, y considerando en la primera únicamente residuos de la producción de quesos.
El volumen de biogás total que se podría generar en la región alcanzaría los $25.652 .242 \mathrm{~m}^{3}$ año ${ }^{-1}$

La cogeneración eléctrica alcanzaría los 71.187 MWh por año. Asimismo, la generación térmica alcanzaría los 74.934MWh al año.

En cuanto a la potencia eléctrica, ésta alcanzaría los 9,0 MW de capacidad instalada, provenientes en su mayor parte de la industria láctea.

La evaluación económica arrojó un potencial económicamente viable para la región de los Ríos de 6,2 MW. Las plantas Colún y Soprole resultaron un VABN positivo, como también frigorífico Valdivia (FRIVAL) y planta Valdivia de la empresa Aguas Décima. No resultó con un VABN positivo el vertedero Morrompulli.

\section{AGRADECIMIENTOS}

Nuestros agradecimientos al Instituto de Ingeniería Agraria y Suelos, Facultad de Ciencias Agrarias, y a los Profesores Teodoro Kausel y Guillaume Serandour, ambos de la Universidad Austral de Chile.

\section{REFERENCIAS}

Altaner, R., 2009. Descripción y análisis comparativo de los sistemas de tratamiento de RIL en la industria de alimentos en Valdivia. Tesis Ingeniero en Alimentos. Universidad Austral de Chile. 167p.

Altuve, J., 2004. El uso del valor actual neto y la tasa interna de retorno para la valoración de las decisiones de inversión. http://www.saber.ula.ve/bitstream/123456789/17341/1/articulo1.pdf (acceso, 05.01.2017).

Centro de Energías Renovables (CER), 2012. Reporte CER, boletín marzo 2013. Resumen anual 2012. http://cer. gob.cl/wp-content/uploads/downloads/2013/03/Reporte-20121.pdf (acceso, 04.08. 2013).

Centro Nacional para la Innovación y Fomento de las Energías Sustentables (CIFES), 2016. Reporte CIFES, Energías Renovables en el Mercado Eléctrico Chileno. Julio, 2016. http://cifes.gob.cl/documentos/reportes-cifes/ reporte-cifes-julio-2016/ (acceso, 05.01.2017).

Comino, E., Riggio, V., Rosso, M., 2012. Biogas production by anaerobic co-digestion of cattle slurry and cheese whey. Bioresource Technology 114, 46-53. http://www.sciencedirect.com/science/article/pii/ S0960852412003410

Comisión Nacional de Energía (CNE), 2013. Precio nudo medio de Mercado Sistema interconectado central, Septiembre 2012 -Diciembre 2012. http://www.cne. cl/tarificacion/electricidad/precios-de-nudo-de-cortoplazo/466-pmm-sic (acceso, 18.03.2013).

Comisión Nacional de Energía (CNE), Deutsche Gesellschaft Technische Zusammenarbeit (GTZ), 2007. Potencial de Biogás. Identificación y clasificación de los principales tipos de biomasa disponibles en Chile para la generación de Biogás. Santiago, Chile.

Comisión Nacional de Energía (CNE), Deutsche Gesellschaft Technische Zusammenarbeit (GTZ), 2009. Las energías 
renovables no convencionales en el mercado eléctrico Chileno. Santiago, Chile.

Comisión Nacional de Energía (CNE), Deutsche Gessellschaft Für Internationale Zusammenarbeit (GIZ), 2012. Guía de Planificación para proyectos de Biogás en Chile. Santiago, Chile.

Comisión Nacional de Energía (CNE), Gamma Ingenieros, 2010. Estudio "análisis estratégico de elementos de política para biogases combustibles en Chile”. N 610-48le09. http://antiguo.minenergia.cl/minwww/export/ sites/default/05_public_Estudios/descargas/estudios/ RESUMEN_EJECUTIVO_ELEMENTOS_POLITICA_BIOGASES.pdf (acceso, 25.07.2013).

Deublein, D., Steinhauser, A., 2008. Biogas from waste and renewable resources, an introduction. WILEY-VCH Verlag GmbH \& Co. KGaA, Weinheim.

Eltawil, M., Belal, E., 2009. Evaluation and scrubbing of biogas generation from agricultural wastes and water hyacinth. Biological Engineering. Misr Journal of Agricultural Engineering 26(1), 534-560. http://www.mjae.eg.net/ pdf/2009/jan/29.pdf

Flotats, X., Campos, E., Bonmati, A., 1997. Aprovechamiento energético de residuos ganaderos. Departamento de medio ambiente y Ciencias del suelo. Universidad de Lleida. http://web.udl.es/usuaris/lea/archivos\%20 pdf/Apunts.pdf (acceso, 10.08.2013).

Gamma Ingenieros S.A., 2011. Modelos de negocio que rentabilicen aplicaciones de biogás en Chile y su fomento. Ministerio de Energía, Chile.

Gil, M., Celma, M., 2003. Concepto, contenido y objeto de la administración de empresas. Editorial UOC Papers, Barcelona, España.

Gobierno Regional de los Ríos, 2012. Manejo sustentable de residuos sólidos. Relleno sanitario de Valdivia, Estimación de residuos. http://seia.sea.gob.cl/archivos/Estimacion_re siduos.pdf (acceso, 15.08.2013).

Hall, S., Roman, R., Cuevas, F., Sánchez, P., 2009. ¿Se necesitan represas en la Patagonia? Un análisis del futuro energético Chileno. Universidad de Chile. Edición Ocho Libros Editores. Santiago, Chile.

Iglinski, B., Buczkowski, R., Iglinska, A., Cichosz, M., Piechota, G., Kujawski, W., 2012. Agricultural biogas plants in Poland: investment process, economical and environmental aspects, biogas potential. Renewable and Sustainable energy reviews 16(7), 4890-4900.

Institut Für Energetik Und Umwelt (IEU), 2006. Biogas gewinnung und nutzung. Handreichung. 3., Auflage. Gülzow, Germany.

Instituto Nacional de Estadística (INE), 2008. Distribución y consumo energético en Chile. http://www.ine.cl/canales/sala_prensa/archivo_documentos/enfoques/2008/ septiembre/energia_pag.pdf (acceso, 05.07.2013).

Instituto Nacional de Estadística (INE), 2011. Generación eléctrica por regiones. http://www.ine.cl/canales/chile_ estadistico/estadisticas_economicas/energia/series_estadisticas/series_estadisticas.php (acceso, 05.05.2012).

Instituto Nacional de Estadística (INE), 2012. Estadísticas del medio ambiente. Compendio estadístico 2012. http:// www.ine.cl/canales/menu/publicaciones/compendio_ estadistico/pdf/2012/medios_ambientales.pdf (acceso, 13.11.2012).
Instituto Nacional de Estadística (INE), 2013. Anexos al informe final. Comisión externa revisora del censo 2012. http://www.censo.cl/anexos_comision_externa_revisora.pdf. (acceso, 30.08.2013).

Instituto Nacional de Estadística (INE), 2015. Boletín Informativo del Instituto Nacional de Estadísticas. Edición $N^{\circ} 52$. http://www.inelosrios.cl/archivos/files/pdf/Coyunturales /2016/2/Bolet\%C3\%ADn_Energ\%C3\%ADa_Diciembre_2015.pdf (acceso, 05.01.2017).

Kaltschmitt, M., Themelis, N., Bronicki, L., Söder, L., Vega, L., 2013. Renewable Energy Systems. Springer-Verlag. New York, USA.

Martin, J., 2007. Un protocolo para la cuantificación y presentación de informes del comportamiento de los sistemas de digestión anaeróbica del estiércol de ganado. Simposio internacional sobre la calidad del aire y residuos. http://metanoamercados.org/recursos/Protocolo_sistemas_anaerobicas_AgStar.pdf (acceso, 05.09.2013)

Martin, J., Coombe, J., Henn, K., 2012. Dairy cattle mortality management via anaerobic digestion. http://northeast. manuremanagement.cornell.edu/Pages/General_Docs / Events $/$ Final.Proceedings.Document.pdf\#page=90 (acceso, 04.01.2017).

Massé, D.L., Masse, L., Hince,J. F., Pomar, C., 2008. Psychrophilic anaerobic digestion biotechnology for swine mortality disposal. Bioresource Technology 99(15), 73.

Ministerio de Energía, 2013. Fijación precios nudo de electricidad. Diario Oficial. http://www.minenergia.cl/ archivos_bajar/2013/D_107_12-02-13.pdf. (acceso, 15.03.2013).

Monárdez, P., Acuña, H., Scott, D., 2008. Evaluation of the potential of wave energy in Chile. $27^{\text {th }}$ International Conference on Offshore Mechanics and Arctic Engineering, ASME, Estoril, Portugal.

Oficina De Estudios y Políticas Agrarias (ODEPA), 2013. Boletín de la leche, avance 2013. http://www.odepa.gob.cl/ menu/MacroRubros.action;jsessionid=C3D0865CBA24 D79ED6E21794EC022E8B?rubro=lactea\&reporte=ava nce_mensual. (acceso, 21.03.2013).

Pavez, A., 2013. Calefacción distrital por biomasa, calor centralizado y sustentable. Sustentabit. Edición 16 de marzo: 10-15. http://www.energiadelsur.com/pdf/Biomada-Revista-BIT.pdf (acceso, 15.03.2013).

Prazeres, A., Carvalho, F., Rivas, J., 2012. Cheese whey management: A review. Journal of Environmental Management 110, 48-68. http://www.sciencedirect.com/science/article/pii/S0301479712002769

Quesada, R., Salas, N., Arguedas, M., Botero, R., 2007. Generación de energía eléctrica a partir de biogás. Tierra Tropical 3(2), 139-147.

Santos, E., 1983. Utilización de diferentes niveles de proteína en combinación con suero de queso en cerdos en crecimiento y acabado. Tesis magíster scientiae. Universidad Nacional de Colombia. 83p.

Schievano, A., D'imporzano, G., Adani, F., 2009. Substituting energy crops with organic wastes and agro-industrial residues for biogas production. Journal of Environmental Management 90(8), 2537-2541.

Soto, I., Werner, L., 2009. Evaluación técnica y económica de una central termoeléctrica en la región de los ríos. Tesis Ingeniero Mecánico. Valdivia. Universidad Austral de 
Chile. Facultad de Ciencias de la Ingeniería. 106p.

Servicio de Evaluación Ambiental (SEIA), 2005. Declaración de impacto ambiental proyecto "sistema de tratamiento RILES frival S.A.". http://seia.sea.gob.cl/expediente/ ficha/fichaPrincipal.php? modo=ficha\&id_expediente $=988939$ (acceso, 20.11.2012).

Servicio de Evaluación Ambiental (SEIA), 2012. Proyectos en carpeta. http://seia.sea.gob.cl/busqueda/buscarProyectoAction.php? modo=ficha\&nombre $=$ carbon\&sector
$=\&$ regiones $=2420135 \&$ presentacion=undefined $\&$ busca $r=$ true (acceso, 25.01.2012).

Zanelli, J., Antonijevic, I., Baeza, R., Echeverria, C., Gambardella, C., Infante, P., Jadresic, A., Jofre, A., Stutzin, A., Tenreiro, C., Martinez, C., Mora, G., 2008. La opción núcleo-eléctrica en Chile. Comisión Chilena de Energía Nuclear. Santiago, Chile. Zaror, C., 2000. Introducción a la Ingeniería Ambiental para la industria de procesos. Universidad de Concepción, Concepción, Chile. 